\title{
The Correlation Between Availability of Pediatricians and Child Mortality within the United States
}

\author{
Catalina Luna $^{1}$, Lauren Stone ${ }^{2}$, Diana Feier ${ }^{3}$, Tyler Shroll ${ }^{4}$ \\ ${ }^{1}$ Department of Chemistry, ${ }^{2}$ Department of Biology, ${ }^{3}$ Department of Mechanical and Aerospace \\ Engineering
}

Faculty Mentor: Anne Donnelly, Center for Undergraduate Research

\begin{abstract}
As the profession of pediatrics becomes more popular, the question of how necessary pediatricians are becomes increasingly prevalent. Understanding whether the availability of pediatricians is correlated to child mortality rates in the United States is essential to provide statistical evidence on the efficacy of current pediatric practices. This study aims to identify a possible correlation between the availability of pediatricians and the mortality rates of children 18 and under in the United States. Data on U.S. child mortality rates from the CDC's WONDER database and data on pediatricians from the American Board of Pediatrics were collected to calculate and examine correlation coefficients. The first test examined the correlation between the ratio of pediatricians in each state with state-by-state child mortality rates in 2018. The second test examined the number of certified pediatricians from 2000-2018 in the entire U.S. population with the child mortality rate in the same time period. The third and fourth tests examined the correlation between the number of certified pediatricians in 2000-2018 in the entire U.S. population with the child mortality rate due to cardiac-related and endocrine-metabolic diseases, respectively. Each test showed a moderate negative correlation demonstrating an implied relationship in which a higher number of pediatricians relates to lower child mortality rates. These results can be used to further public knowledge on the effects and potential benefits that pediatricians have on childhood health and development, helping parents make more informed decisions regarding their child's pediatric care options.

Keywords: Cardiac Disease, Child Mortality, Endocrine-Metabolic Diseases, Pediatricians
\end{abstract}

\section{Introduction}

As the profession of pediatrics becomes more popular, the decision of whether a pediatrician is necessary becomes increasingly prevalent (American Board of Pediatrics, 2020).

Understanding whether the availability of pediatricians is correlated to child mortality rates in the United States is essential to provide statistical evidence on the efficacy of current pediatric practices and allow parents to make informed decisions on potential benefits of taking their child 
to a pediatrician. Based on previous literature, it can be hypothesized that there will be a negative correlation between pediatrician prevalence and childhood mortality.

While previous studies have demonstrated the effect of pediatricians on specific pediatric health concerns, there have been no studies analyzing the impact that having a pediatrician has on the mortality rate of children 18 and under in the United States (Huang et al., 2011; Moss et al., 2015). Previous research has found that for every ten additional primary care physicians per 100,000 people, life expectancy increased 51.5 days (Basu et al., 2019). Given the similar roles of primary care physicians and pediatricians, such research would suggest a negative correlation could exist between pediatricians and mortality rate. This study aims to analyze the extent of this relationship and whether the supply of pediatricians has a significant impact on the mortality rate of nutritional and metabolic diseases. A similar study conducted in Japan found a seven percent decrease in the mortality rate of children under five-years old with every unit increase in pediatrician density (Sakai et al., 2016). The negative correlation between pediatrician population and mortality rate of children under five-years old suggests a possible relationship between pediatrician supply and child mortality in the United States. However, it is common practice in the United States for a child to see a pediatrician up until they are 18 years old. Thus, the present study is focused on the mortality rate of children 18 years old and under.

Research shows that some of the most prevalent causes of mortality in children under 18 years old include cardiovascular disease, influenza, cancer, and drug overdose (Curtin et al., 2017; Heron, 2019). Pediatric obesity has become an increasingly prominent issue in the United States that can have long-lasting health implications (DeBoer et al., 2019). One study comparing the weight care of children between pediatricians and family physicians revealed that pediatricians were more diligent in their assessment of weight in children and more likely to provide behavior counseling (Huang et al., 2011). This suggests that a greater supply of pediatricians could lower the obesity rate in children. Furthermore, one study suggests that a higher density of pediatricians could lead to more HPV vaccinations, thus suggesting that pediatricians could decrease mortality rate through the implementation of vaccinations (Moss et al., 2015).

Previous research indirectly implies that a higher pediatrician population could decrease the mortality rate of children. Therefore, within this study, it is hypothesized that there is a strong, negative correlation between the availability of pediatricians and child mortality within the 
United States. Furthermore, this study seeks to fill the gap by directly analyzing the impact that the pediatrician population has on the mortality rate of children 18 years old and under in order to help the general population and healthcare system determine the effectiveness of pediatricians on pediatric health. Furthermore, this study aims to identify and characterize possible correlation between the availability of pediatricians and the mortality rates of children ages 18 and under in the United States. This study also aims to identify any knowledge gaps in the effect of pediatricians on the general mortality rate and mortality rate caused by specific disease in children 18 and over as well as provide possible future areas of study. Online databases will be used to collect the desired data- distribution of pediatricians and child mortality rates throughout the United States- and a statistical test will be conducted to examine the correlation between the density of pediatricians in the United States and child mortality rates.

\section{Methods}

\section{Data Sources}

The distribution of currently certified pediatricians per 100,000 children in 2018 was collected from the American Board of Pediatrics, as displayed in Figure 1. The map is colorcoded, where blue distinguishes states with higher ratios and yellow indicates states with lower ratios of pediatricians to children. In addition, data on the trend of pediatrician certifications from 1934 to 2020 was collected from the American Board of Pediatrics, as demonstrated in Figure 2. However, only the data from 2000 to 2018 was used in this study. In both of these sources, only the pediatricians certified in general pediatrics, as opposed to subspecialties, were considered in order to define a broader base of pediatric practice. 


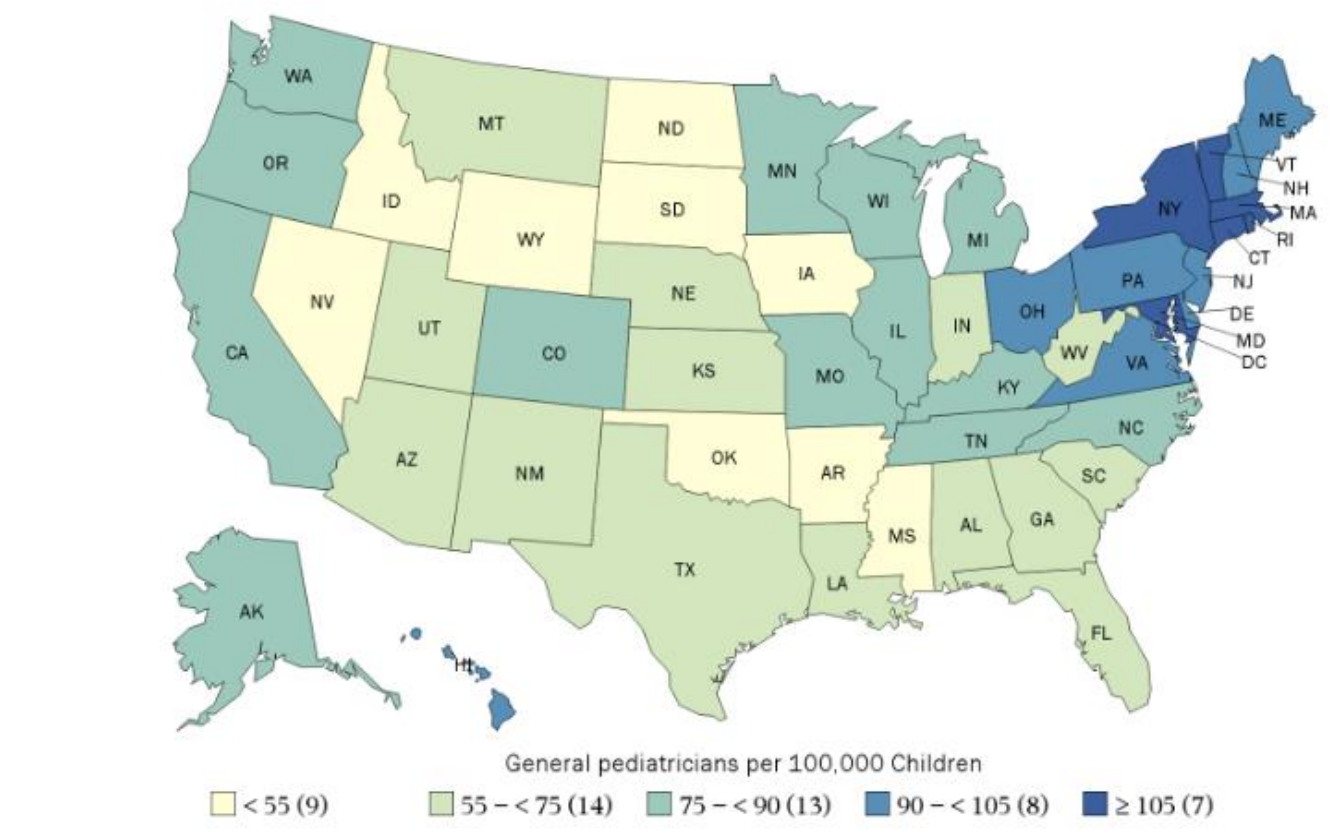

Figure 1. Pediatricians Currently Certified in General Pediatrics per 100,000 Children in 2018, Distinguished by State (American Board of Pediatrics, 2019).

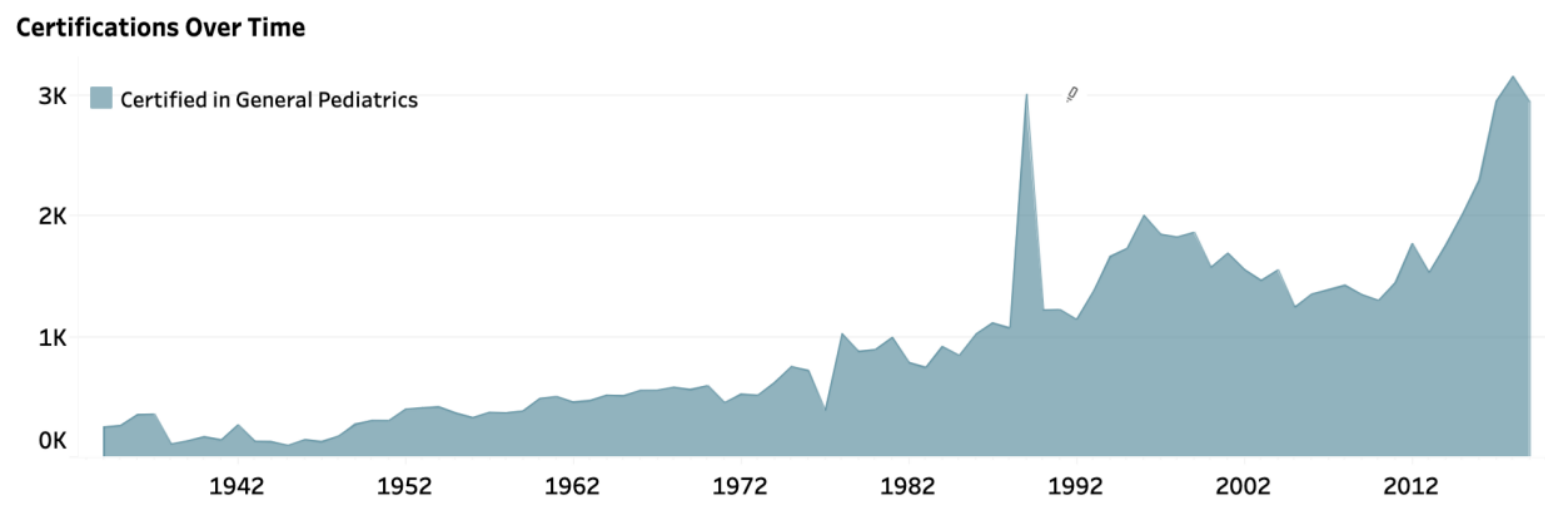

Figure 2. Certifications in General Pediatrics in the United States from 1936 to 2020 (American Board of Pediatrics, 2020).

Mortality rates of children ages 18 and under were collected from the Center for Disease Control and Prevention's WONDER online database (Underlying Cause of Death, 2020). Mortality rates per 100,000 children for the entire United States population were pulled from the years 2000 to 2018 . Mortality rates were specified by mortality from all causes for children ages 18 and under. These rates were then narrowed to particular causes of death, specifically diseases of the circulatory system as well as nutritional and metabolic diseases, for further analysis. 
Furthermore, mortality rates for each state were gathered from the year 2018. Although the CDC included Washington D.C. as a separate entity in their data sets, it was not included in the state-by-state analysis due to the fact that it is defined as a city and thus deviates from the purpose of this study. For this state data collection, mortality rates from all causes of death were included.

\section{Statistical Analysis}

After each set of data was collected, a Pearson product-moment correlation test was run on the data finding the relationship between the following four pairings:
A. The number of pediatricians certified in General Pediatrics per 100,000 Children in 2018 by state and child mortality rates per 100,000 children in 2018 by state.
B. The number of certifications in general pediatrics in the United States from 2000 to 2018 and child mortality rates per 100,000 children from 2000 to 2018.
C. The number of certifications in General Pediatrics in the United States from 2000 to 2018 and child mortality rates due to diseases of the circulatory system per 100,000 children by year from 2000 to 2018 .
D. The number of certifications in General Pediatrics in the United States from 2000 to 2018 and child mortality rates due to endocrine and metabolic diseases per 100,000 children by year from 2000 to 2018 .

Each data pairing was placed in an excel table where the Pearson correlation test was run using the "=CORREL" function in Microsoft Excel. The tables were constructed as such:
A. The 50 states and District of Columbia were in the first column. The number of pediatric certifications were placed in the second column, by state, and the child mortality rates were placed in the third column, by state. The second and third columns were analyzed through the correlation function.
B. The years 2000-2018 were in the first column. The number of pediatric certifications were placed in the second column, by year, and the child mortality rates were placed in the third column, by year. The second and third columns were analyzed through the correlation function.


C. The years 2000-2018 were in the first column. The number of pediatric certifications were placed in the second column, by year, and the child mortality rates due to diseases of the circulatory system were placed in the third column, by year. The second and third columns were analyzed through the correlation function.

D. The years 2000-2018 were in the first column. The number of pediatric certifications were placed in the second column, by year, and the child mortality rates due to endocrine, nutritional and metabolic diseases were placed in the third column, by year. The second and third columns were analyzed through the correlation function.

Afterwards, the relationship between the two datasets in each pairing was analyzed based on the value of the correlation coefficient produced by the Pearson correlation test. This coefficient was used to assess the strength of linear correlation between each data pairing. The following ranges were set prior to obtaining results: if the absolute value of correlation function was 0 0.30 , the sets have no correlation; $0.30-0.50$, the sets have a weak correlation; $0.50-0.70$, the sets have a moderate correlation; and 0.70-1.00 the sets have a strong correlation.

\section{Results}

\section{State Comparison}

Pairing A was conducted to examine the correlation between the number of pediatricians certified in General Pediatrics per 100,000 Children in 2018 by state and child mortality rates per 100,000 children in 2018 by state. Figure 3 depicts a moderate correlation between these pairings with a correlation coefficient of -0.513 . The moderate correlation of this pairing indicates a negative relationship between the number of pediatricians and child mortality rate. The states with a larger ratio of pediatricians per 100,000 children tended to have lower mortality rates. Thus, this data suggests that a higher number of pediatricians per child is consistent with a lower child mortality rate. 
Child Mortality Rate per 100,000 children vs. Ratio of Pediatricians per 100,000 Children in the 50 States in 2018

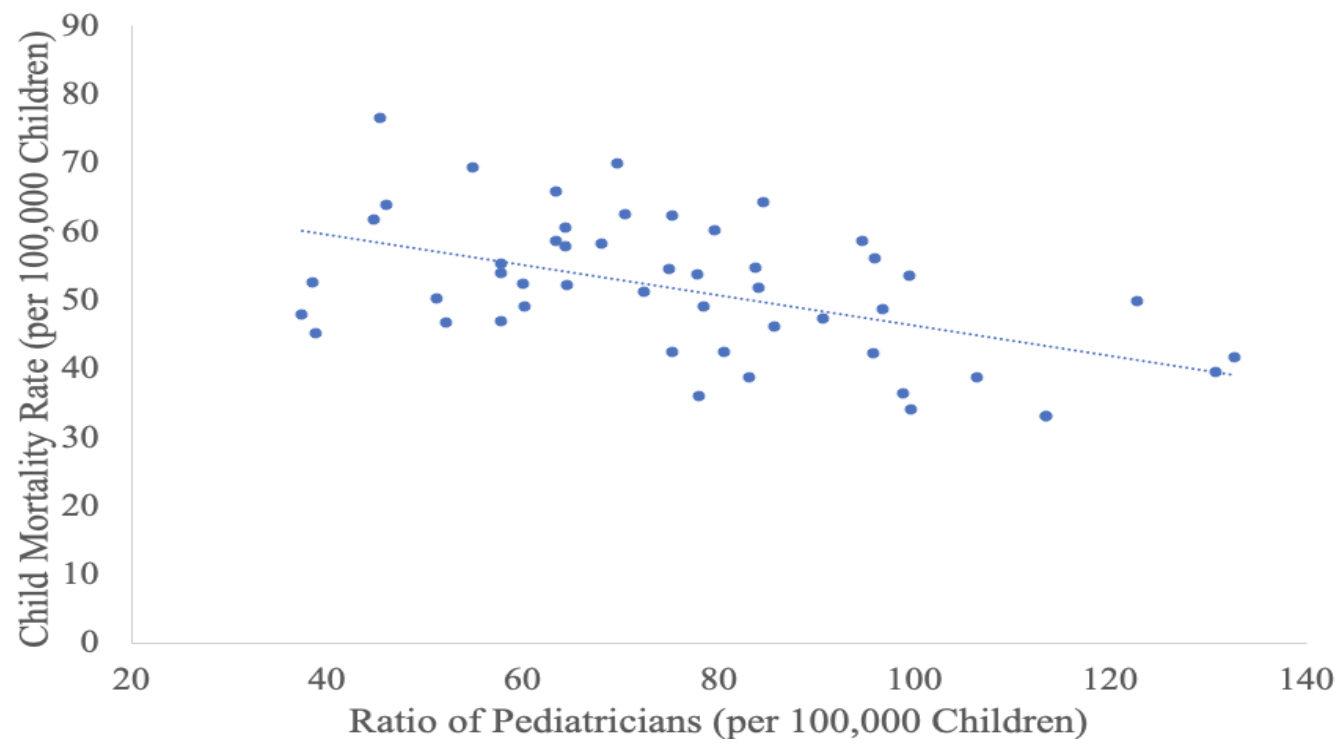

Figure 3. The Correlation Between the Number of Pediatricians Certified in General Pediatrics per 100,000 Children in 2018 by State and Child Mortality Rates per 100,000 Children in 2018 by State.

\section{0-2018 General Comparison}

Figure 4 shows the data for pairing B, the number of certifications in general pediatrics in the United States from 2000 to 2018 and child mortality rates per 100,000 children from 2000 to 2018. A correlation coefficient was calculated between the two sets of data and was found to be 0.559 . Based on the initially established scale, this indicates that there is a negative moderate correlation between the number of certifications in general pediatrics and child mortality rates between 2000 and 2018. In other words, years that had more general pediatricians certified tended to also have lower child mortality rates. 
Child Mortality Rate per 100,000 Children vs. the Number of Certified General Pediatricians in the United States from 2000 to 2018

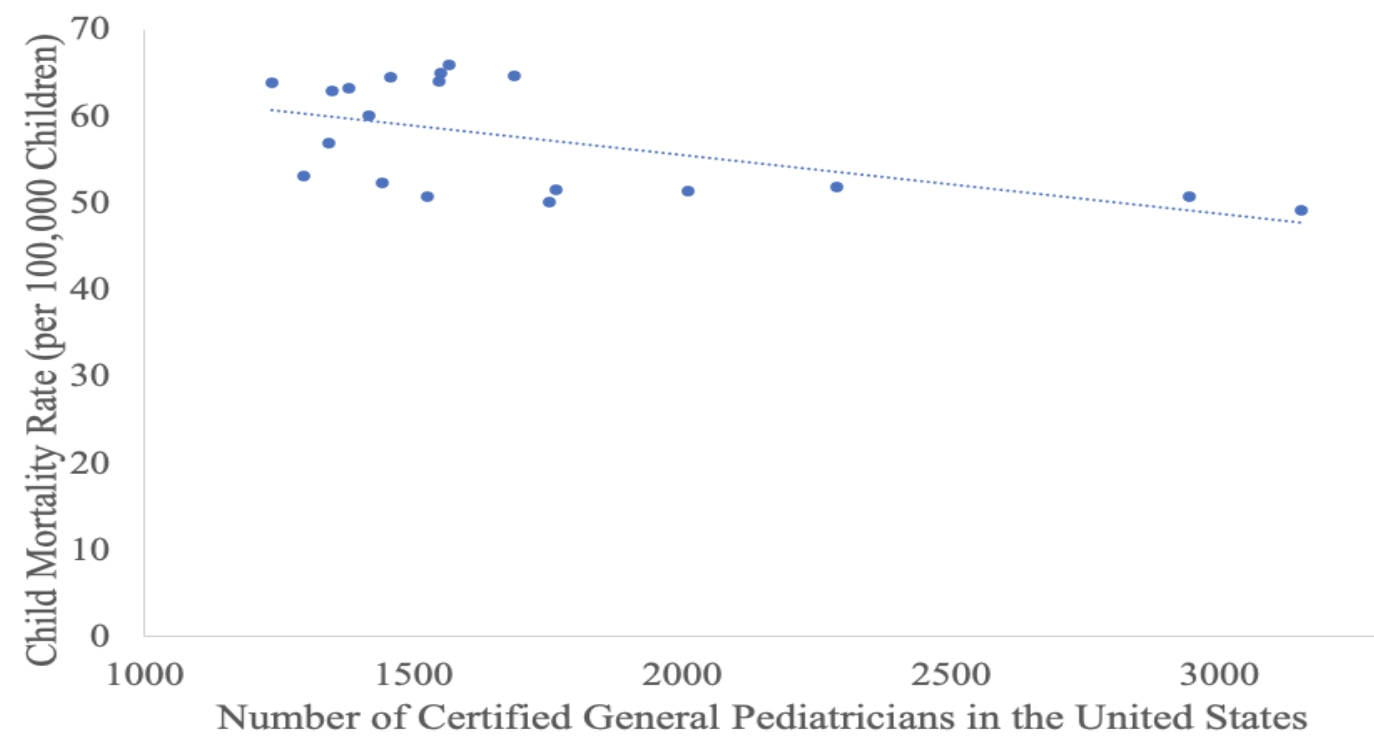

Figure 4. The Correlation Between the Number of Certifications in General Pediatrics in the United States from 2000 to 2018 and Child Mortality Rates per 100,000 Children from 2000 to 2018.

\section{8-2018 Cardiac Diseases Comparison}

The data for pairing $\mathrm{C}$, the number of certifications in general pediatrics in the United States from 2000 to 2018 and child mortality rates per 100,000 children due to cardiac disease from 2000 to 2018, is shown in Figure 5. The correlation coefficient between the two sets was found to be -0.537 . This coefficient suggests a moderate negative correlation between the number of general pediatricians and child mortality rates due to cardiac disease between 2000 and 2018 . This means that years in which there were more general pediatricians certified also tended to have lower child mortality rates due to cardiac disease. 
Child Mortality Rate Due to Cardiac Diseases per 100,000

Children vs. the Number of Certified General Pediatricians

in the United States from 2000 to 2018

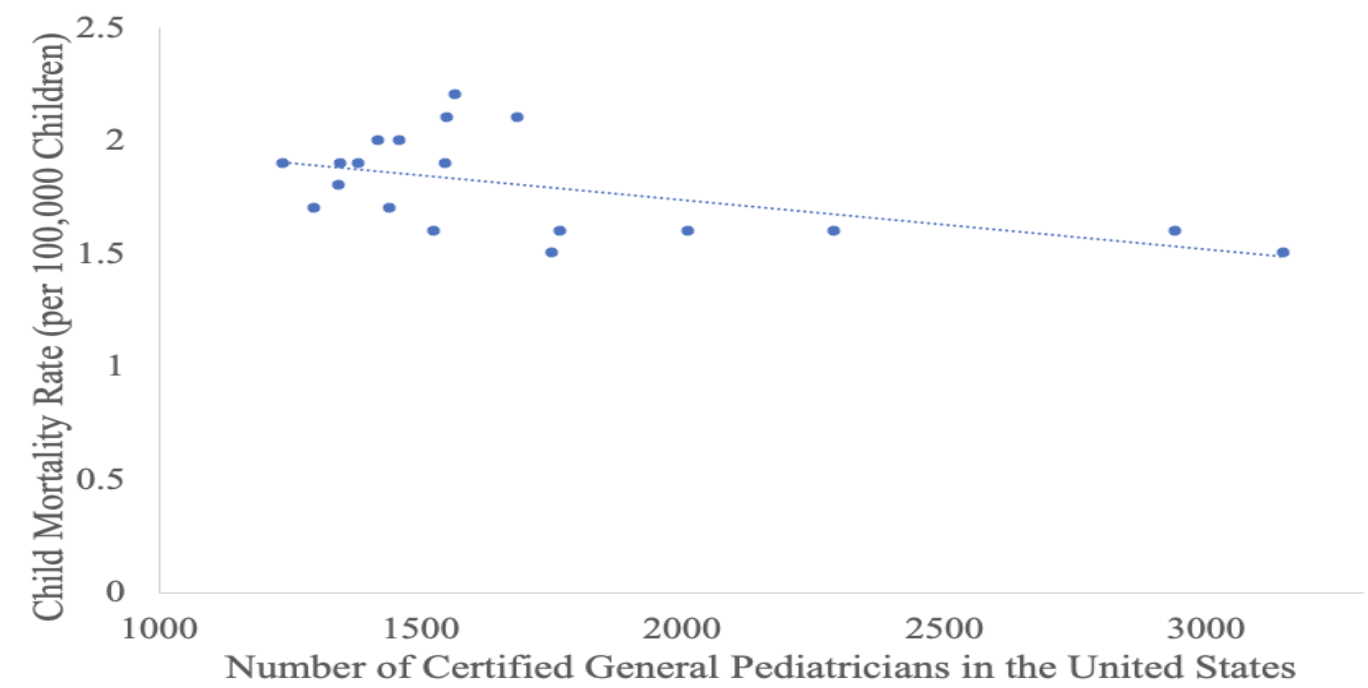

Figure 5. The Correlation Between the Number of Certifications in General Pediatrics in the United States from 2000 to 2018 and Child Mortality Rates per 100,000 Children due to Cardiac Disease from 2000 to 2018.

\section{8-2018 Metabolic Diseases Comparison}

The data for pairing $\mathrm{D}$, the number of certifications in general pediatrics in the United States from 2000 to 2018 and child mortality rates per 100,000 children due to endocrine and metabolic diseases from 2000 to 2018, is shown in Figure 6. Figure 6 depicts a moderate, negative correlation of -0.536 between the two variables, suggesting that the years in which there were higher numbers of general pediatricians there tended to also be lower child mortality rates due to endocrine and metabolic diseases. 
Child Mortality Rate Due to Endocrine and Metabolic Diseases per 100,000 Children vs. the Number of Certified General Pediatricians in the United States from 2000 to 2018

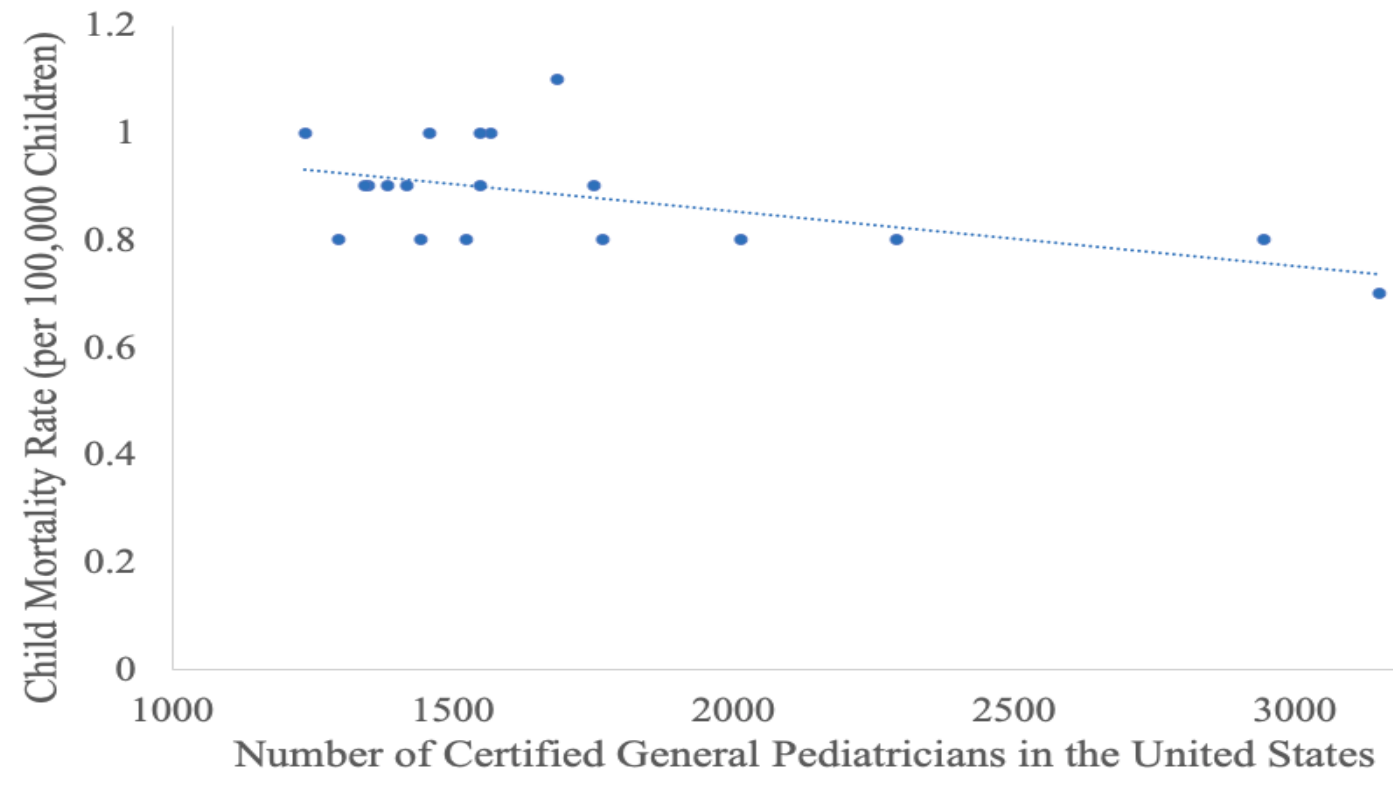

Figure 6. The Correlation Between the Number of Certifications in General Pediatrics in the United States from 2000 to 2018 and Child Mortality Rates per 100,000 Children due to Endocrine and Metabolic Disease from 2000 to 2018.

\section{Discussion}

For all four analysis pairings, the correlations between presence of pediatricians and child mortality rates were found to be moderately negative. In other words, there is an implied link between the availability of pediatricians and a decrease in child mortality rates, indicating that seeing a pediatrician could positively impact the health of children. This correlation was found across a range of 18 years, from 2000 to 2018, and within the 50 states in the year 2018. Since this correlation was found within two separate analyses, there is a stronger implication of the importance of pediatricians in preventing child mortality. Furthermore, the moderate negative correlation was found for endocrine, nutritional and metabolic diseases and diseases of the circulatory system, indicating that pediatricians could have direct impacts on varying aspects of children's health. Overall, although there is not a causal relationship established based on the correlation tests conducted, there is an indicated relationship between pediatrician presence and lower child mortality rates within multiple areas.

These results can be used to further public knowledge on the effects and potential benefits that pediatricians have on childhood health and development. This could help parents make more 
informed decisions regarding their child's pediatric care options, which remains important as skepticism of health care practitioners remains a prevalent issue (Bezreh et al., 2011). These results do not address what causes of death are out of the control of pediatric specialists and the specific role pediatricians play in helping avoid preventable causes of childhood death. Thus, future research could be conducted towards pediatric influence on specific causes of child mortality beyond the causes looked at in this study, as well as how other types of health officials play a role in U.S. child mortality rates.

The results of the study did not completely support the hypothesis, which stated that there would be a strong, negative correlation between the availability of pediatricians and the mortality rates of children ages 18 and under in the United States for each established pairing. Yet the hypothesized relationship was still followed, as the results for each pairing produced moderate negative correlation coefficients. Although the hypothesis was correct in stating the negative relationship between the two variables for each pairing, it was incorrect in regard to the strength of the relationship. Hence, through this statistical test, the extent of each relationship and whether the supply of pediatricians has a significant impact on the mortality rate of nutritional and metabolic diseases was analyzed.

The results of this study are consistent with the findings of other studies in this field. Sakai et al., 2016 reported similar findings, suggesting significant decrease in the mortality rate of children under five-years old with every unit increase in pediatrician density. This study expands upon that previous research by suggesting that the population density of pediatricians is correlated with a decrease in mortality rate of children 18 years-old and under. Furthermore, this study supports the findings of similar research demonstrating that higher supplies of primary care physicians decrease mortality rates. Basu et al., 2019 reports that more primary care physicians were correlated with a significant increase in life-expectancy. As pediatricians and primary care physicians serve similar roles, with pediatricians specialized on children 18 and under, the findings of this research further support the results of Basu et al., 2019. The findings reported in this research can be added to this congregate of studies to suggest that a higher concentration of pediatricians is correlated with a lower child mortality rate. 


\section{Conclusion}

After analyzing data from the CDC Wonder Database and American Board of Pediatrics, the relationship between the availability of pediatricians and child mortality rates was found to have a moderate negative correlation. This finding supports the hypothesis that higher pediatrician numbers are negatively associated with child mortality. Each of the four pairings produced moderate negative correlation coefficients. With this knowledge that seeing a pediatrician is related to lower child mortality rates, parents can make more educated decisions on taking care of the health of their children. Further research needs to be conducted on the exact roles pediatricians have on preventing child mortality and how issues besides child mortality rates are affected by health officials.

\section{Acknowledgements}

We would like to thank Dr. Anne Donnelly, Professor Jennifer Moses, and Zinia Basil for their advice and guidance throughout this study.

\section{References}

American Board of Pediatrics, Pediatric Physicians Workforce Data Book, 2018-2019, Chapel Hill, NC: American Board of Pediatrics, 2019.

Basu, S., Berkowitz, S. A., Phillips, R. L., Bitton, A., Landon, B. E., \& Phillips, R. S. (2019). Association of Primary Care Physician Supply With Population Mortality in the United States, 2005-2015. JAMA Intern Med, 179(4), 506-514. https://doi.org/10.1001/jamainternmed.2018.7624 -+

Bezreh, T., Laws, M. B., Taubin, T., Rifkin, D. E., \& Wilson, I. B. (2012). Challenges to physicianpatient communication about medication use: a window into the skeptical patient's world. Patient preference and adherence, 6, 11-18. https://doi.org/10.2147/PPA.S25971

Curtin, S. C., Tejada-Vera, B., \& Warner, M. (2017, August 16). Products - Data Briefs - Number 282 August 2017. Centers for Disease Control and Prevention. https://www.cdc.gov/nchs/products/databriefs/db282.htm

DeBoer, M. D., Filipp, S. L., \& Gurka, M. J. (2019). Geographical variation in the prevalence of obesity and metabolic syndrome among US adolescents. Pediatric Obesity, 14(4), e12483. https://doi.org/10.1111/ijpo.12483

Heron M. (2019). Deaths: Leading Causes for 2017. National vital statistics reports: from the Centers for Disease Control and Prevention, National Center for Health Statistics, National Vital Statistics System, 68(6), 1-77. 
Huang, T. T., Borowski, L. A., Liu, B., Galuska, D. A., Ballard-Barbash, R., Yanovski, S. Z., Olster, D. H., Atienza, A. A., \& Smith, A. W. (2011). Pediatricians' and family physicians' weight-related care of children in the U.S. American Journal of Preventive Medicine, 41(1), 24-32. https://doi.org/10.1016/j.amepre.2011.03.016

Moss, J. L., Reiter, P. L., \& Brewer, N. T. (2015). Correlates of human papillomavirus vaccine coverage: a state-level analysis. Sexually Transmitted Diseases, 42(2), 71-75. https://doi.org/10.1097/OLQ.0000000000000225

Pediatricians Ever Certified by the ABP Since 1934. (2020). The American Board of Pediatrics. https://www.abp.org/content/pediatricians-ever-certified-1934

Sakai, R., Fink, G., Kumamaru, H., \& Kawachi, I. (2016). The Impact of Pediatrician Supply on Child Health Outcomes: Longitudinal Evidence from Japan. Health Services Research, 51(2), 530-549. https://doi.org/10.1111/1475-6773.12354

Underlying Cause of Death 1999-2019 on CDC WONDER Online Database. (2020). CDC WONDER Online Database. 